\title{
Effect of Compressive Load on Magnetic Shape Memory Effect in Ni-Mn-Ga Single Crystal
}

\author{
K. Richterováa ${ }^{a}$, J. Drahokoupil ${ }^{b, *}, \mathrm{~V}$. KopeckÝ ${ }^{a}, \mathrm{~K}$. Dragounová ${ }^{b}$, \\ M. LANDA ${ }^{c}$ AND O. HECZKO ${ }^{a}$ \\ ${ }^{a}$ Institute of Physics ASCR, Na Slovance 1999/2, 18221 Praha 8, Czech Republic \\ ${ }^{b}$ Faculty of Nuclear Sciences and Physical Engineering of CTU in Prague, \\ Trojanova 13, 12000 Praha 2, Czech Republic \\ ${ }^{c}$ Institute of Thermomechanics ASCR, Dolejškova 5, 18200 Prague 8, Czech Republic
}

\begin{abstract}
We investigated the effect of large compressive stress on magnetic shape memory effect in modulated $10 \mathrm{M}$ martensite of $\mathrm{Ni}-\mathrm{Mn}-\mathrm{Ga}$ Heusler alloy. The single crystalline sample deformed approximately elastically up to highest load $540 \mathrm{MPa}$. Structural and microstructural changes of a single crystal were analysed by X-ray diffraction $2 \mathrm{D}$ scan mapping in $2 \theta$ and $\omega$. Although the crystal structure, lattice cell parameters, and twinned microstructure ( $a / b$ and modulation twinning) exhibited only small changes after the loading, the volume of the sample undergoing the magnetically induced reorientation decreased sharply while magnetic field necessary for the reorientation gradually increased. The extrapolation suggested that no magnetically induced reorientation might occur after compression of about $1 \mathrm{GPa}$.
\end{abstract}

DOI: $10.12693 /$ APhysPolA.128.704

PACS: $61.05 . c p, 62.40 .+\mathrm{i}, 75.50 . \mathrm{Cc}, 75.80 .+\mathrm{q}$

\section{Introduction}

Magnetic shape memory (MSM) effect was discovered by Ullakko in 1996 when he observed large magnetic field induced deformation $(0.3 \%)$ in $\mathrm{Ni}-\mathrm{Mn}-\mathrm{Ga}$ single crystal [1]. Maximum deformation reported to date in $\mathrm{Ni}-\mathrm{Mn}-\mathrm{Ga}$ is about $12 \%$ which is two orders of magnitude higher than giant magnetostriction $(0.2 \%)$ in comparable (weak) magnetic field below $1 \mathrm{~T}$ or $10 \mathrm{kOe}$ [2]. The mechanism of the deformation is the reorientation of low symmetry crystal structure by movement of twin boundaries [1, 3]. The effect depends on the twinned microstructure of the low symmetry martensitic phase and high mobility of twin boundaries, which move through the crystal without large impediment. Martensitic transformation from parent cubic austenite phase to lower symmetry martensite phase enables twinning in the martensite phase $[3,4]$. The reorientation by twin boundary motion can occur under mechanical stress or magnetic field. The twin variant with easy axis along the external force grows on the account of other differently oriented variants [5]. As the giant deformation is caused by reorientation, the MSM effect is also called magnetically induced reorientation (MIR) [3].

In $\mathrm{Ni}-\mathrm{Mn}-\mathrm{Ga}$, the cubic austenite phase transforms to various martensitic phases depending on the composition [6]. It is usually the modulated phase, which exhibits extremely high mobility of twin boundaries $[7,8]$. Here we focus on modulated $10 \mathrm{M}$ phase which exhibits

\footnotetext{
* corresponding author; e-mail: jan.drahokoupil@fji.cvut.cz
}

$6 \%$ deformation. The structure can be approximated by tetragonal structure with slight monoclinic distortion [3]. The short crystal axis, $c$-axis, is also magnetic easy axis, i.e. the magnetization prefers $c$-direction and material is magnetically anisotropic. Due to the monoclinicity, there are various twinning systems, $a-b$ and $a-c$ twinning, monoclinic twinning $[9,10]$. The MIR can occur only by movement of $a-c$ twin boundaries as variant with $c$-axis along field is preferred. The mobile $a-c$ twin boundary is macro-interface with rich internal structure of monoclinic and $a-b$ twinning on microscale [11].

As the magnetic field is a relatively weak force, the existential condition for the effect is extremely low twinning stress or extremely high mobility of twin boundaries. Additionally for the existence of the effect it is necessary that new variant with mobile boundaries can nucleate easily. It was observed that the field needed to nucleate the twin boundary, i.e. when starting from single variant, is usually somehow higher than field needed to move twin boundary $[12,13]$.

It was shown that various magnetomechanical training can significantly decrease the twinning stress. The training consists of repeated compression along various axes and rotation in magnetic field $[13,14]$. This training apparently establishes favourable microstructure, although the mechanism how it affects the motion, is not quite clear. Surely the training helps to form twin boundaries arrangement in which moving boundaries do not block each other [15].

The high mobility can be secured only in single crystal as grain boundaries effectively block any twin boundary motion [16]. The extreme high mobility is also connected with perfection of the crystal, since any imperfection can 
hinder the twin boundary motion [17]. Moreover, it was shown that different types of $a-c$ twin boundaries, arising due to monoclinic distortion, exhibit sharply different mobility [9].

Here we study an inverse problem. We have more or less "perfect" material, which exhibits full structural reorientation in very low magnetic field (or twinning stress), i.e. the magnetic field switches the single variant with $c$-axis perpendicular to field to variant with $c$-axis along the field. The aim is to find how the lattice deformation and microstructure changes induced by very large mechanical stress (in comparison with twinning stress) influence the nucleation of new twins and twin boundary mobility in a hope to shed some light on the mechanism of MIR or twin nucleation and following twin boundary motion.

\section{Experimental}

Rectangular samples with faces approximately along $\{100\}$ planes of cubic austenite were cut from large single crystalline ingot grown by modified Bridgman method. The approximate composition was $\mathrm{Ni}_{50} \mathrm{Mn}_{29} \mathrm{Ga}_{21}$. The ingot was heat treated for homogenization and ordering $[13,18]$. The size of investigated sample was $0.95 \mathrm{~mm} \times 2.5 \mathrm{~mm} \times 6.8 \mathrm{~mm}$. Transformation from austenite to martensite occurred at about 326 K. Ferromagnetic transition or the Curie temperature was $373 \mathrm{~K}$. At room temperature the sample had $10 \mathrm{M}$ structure, which could be approximately described as monoclinic with $a=0.5973 \mathrm{~nm}, b=0.5951 \mathrm{~nm}, c=0.5586 \mathrm{~nm}$, $\gamma=90.36 \mathrm{deg}$

To evaluate the influence of the compression stress, the sample was compressed along short dimension of rectangle up to $540 \mathrm{MPa}$, which was the limit of our setup. This compression geometry is not very usual for compression testing but this arrangement was needed for investigating magnetically induced reorientation (MIR) and to observe twin boundary movement. After compression, the sample was in single variant state with $c$-axis aligned with the load axis (Fig. 1). Electromechanical test machine Tira2300 (Germany), with miniature LVDT transducer type CD375 (MacroSensor, USA) for a precise measurement of die displacement was used to apply the compression stress. The die was made of (hard) cemented carbides. Since the geometry was not very suitable and the sample was small, the stress-strain curves had to be carefully corrected for machine deformation.

After each compression, the magnetization curve of the sample with the magnetic field perpendicular to the compression direction was measured. The sample was magnetized along the longest sample dimension to minimize the effect of demagnetization. From measured magnetization curve we evaluated the parameters characteristic for MIR, the nucleation field, i.e. the field needed to nucleate new twin variant with $c$-axis along the field direction and transformation volume i.e. the volume of residual variant which did not reorient in the field, i.e. the variant with $c$-axis along the compression stress. The magnetization

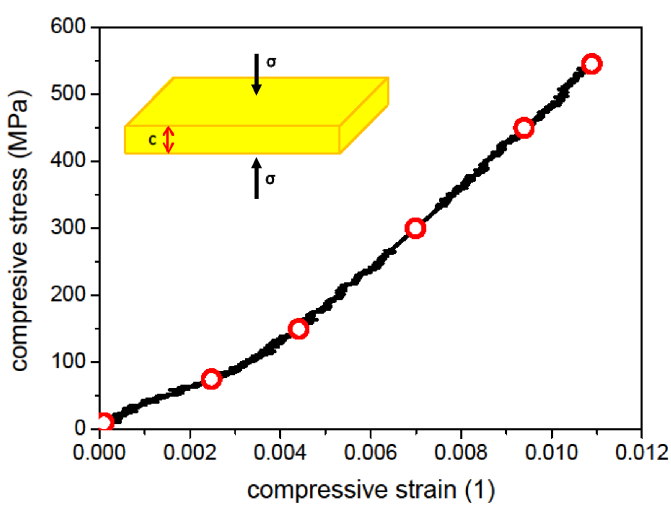

Fig. 1. Compressive stress-strain curve of Ni-Mn-Ga sample. The red circles marked the point where the compression was stopped and MIR and X-ray analysis on unloaded sample was done. The inset shows the geometry of loading.

curves were measured by vibrating sample magnetometer. After this first measurement, the sample was again magnetized along short dimension to obtain the initial orientation of $c$-axis as after the compression. The measured magnetization curve proved that final state was again single variant. This procedure secured consistent analysis of structure and microstructure by X-ray. We repeated this measuring procedure for gradually increased compressive stress up to $545 \mathrm{MPa}$. After each compression and after magnetization measurements (MIR measurement) the detailed X-ray analysis of martensitic structure and microstructure was performed.

The XRD measurement was made on Panalytical diffractometer X'Pert PRO with Co tube $(\lambda=$ $0.1789 \mathrm{~nm}$ ). The parallel beam geometry with parabolic multilayer mirror in primary beam and parallel plate collimator (divergence $0.06^{\circ}$ ) in diffracted beam was used. The texture cradle (ATC-3) allows inclining and rotating with the sample. To obtain information about changes of structure and microstructure of the crystals, we measured two-axes diffraction maps $(2 \theta, \omega)$ of lattice planes $(400)$, (040), (004), (600), (060), (440) and (4-40) in different orientations, see below. Using the first set of planes (primary diffraction lines with sum of indexes $=4 n$ with high intensity), we can calculate directly unit cell parameters $a, b$, and $c$. The planes (600), (060) are superstructure lines of the same order and thus their intensity is low but better resolution is obtained [19]. The planes (440) and (4-40) were measured to obtain best resolution for $\gamma$ if $h=k$ and $h, k \neq 0$ according to condition deduced from lattice distance formula of monoclinic cell

$$
\frac{1}{d_{h k l}^{2}}=\frac{\frac{a^{2}}{h^{2}}+\frac{b^{2}}{k^{2}}-\frac{2 h k \cos \gamma}{a b}}{\sin ^{2} \gamma}+\frac{l^{2}}{c^{2}} .
$$

Measured $2 \theta$ scans were fitted for every omega by the Pearson VII function

$$
\begin{gathered}
I(\theta)=\frac{A_{1}}{\left(1+A_{3}\left(2 \theta-A_{2}\right)^{2}\right)^{A_{4}}} \\
+\frac{\bar{A}_{1}}{\left(1+\bar{A}_{3}\left(2 \theta-\bar{A}_{2}\right)^{2}\right)^{A_{4}}},
\end{gathered}
$$


where $A_{1}$ stands for intensity of the peak, $A_{2}$ is the position in $2 \theta, A_{3}$ is the width of the peak and $A_{4}$ is shape parameter, for $A_{4}$ equal to 1 the function becomes the Cauchy function, while for $A_{4}=\infty$ it is Gaussian. Striped quantities $\bar{A}$ mark the same values for the $K_{\alpha_{2}}$ line of the $\mathrm{X}$-ray spectra. If the $\omega$ is not exactly in diffraction condition, there is an instrumental reason for a small shift of $2 \theta$ position. In order to find precisely the diffraction maximum the intensity was fitted by parabola in $\omega$ direction. Resulting positions of the diffractions were compared with their calculated values from our model of unit cell. The lattice parameters of the model were then modified using the least squares method using all measured diffractions.

\section{Results and discussion}

Figure 1 shows a compression stress-strain curve up to maximum stress available in our setup. The stress-strain response was nearly linear, fully reversible and without significant hysteresis indicating that the sample was deformed mostly elastically. There is some change of the slope in the onset most likely due to misalignment of the grips and sample faces. The effect was pronounced as the sample dimension was very small. Marked points on the curve indicate the compression stresses, where the load was stopped and the sample unloaded. Then the X-ray analysis and the measurement of MIR were performed.

The sample was deformed always along the crystallographic $c$-axis. This deformation might seem very special but in fact it is fully general and only possible due to the easiness of twin reorientation. Very small stress, in order of few MPa (twinning stress) is sufficient to reorient the sample to variant with $c$-axis along the stress. Therefore after compression, the $c$-axis is always aligned with the load axis. Such sample was then analysed by X-ray using diffraction from different sides of the sample.

To obtain the reorientation, the sample was magnetized perpendicularly to the $c$-axis. The increasing magnetic field nucleates the variant with the $c$-axis along the field and this variant grows by moving twin boundaries resulting in structure reorientation (MIR). In ideal case, whole sample reorients to single variant with $c$-axis along the field. This ideal case occurred only in our "perfect" sample before compression loading. The gradual increase of the compressive stress and deformation affected the MIR in unloaded sample is summarized in Figs. 2 and 3. They show that the nucleation field, in which the new twin boundaries appears and start moving, is for small compression stresses nearly constant and for higher stresses starts to grow (Fig. 2) while the reoriented volume of the sample or MIR decreases continually (Fig. 3). This indicates that the mobility of twin boundaries gradually decreases and their mobility is influenced already by relatively small compression prior this measurement $(<100 \mathrm{MPa})$. However, in spite of these significant changes in MIR in unloaded sample, there are no significant changes in compression stress-strain curve (Fig. 1). Within measuring precision the material seems to deform elastically up to highest load.

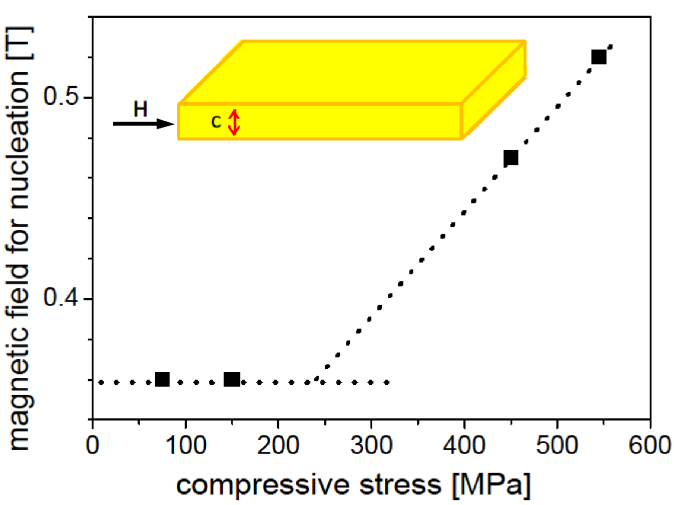

Fig. 2. Magnetic field needed for nucleation of new twin variant along the field in the unloaded sample as a function of previously applied compressive stress. The inset shows field and the sample orientation.

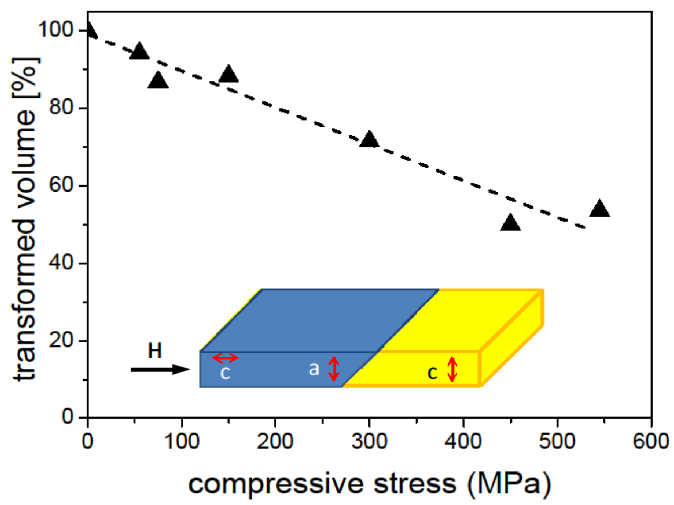

Fig. 3. The volume of transformed variant obtained in magnetic field as a function of previously applied compressive stress. The inset shows schematically two martensitic variants with different orientation joined by mobile twin boundary.

To find what structural and microstructural changes occurred during compression, we performed detailed 2D $\mathrm{X}$-ray studies. Thanks to used mapping method, we were not only analysing the lattice structure but we could catch also more subtle changes in the microstructure of twinned single crystal. To secure consistency in analysing the microstructure, the crystal orientation was always the same consisting of approximately single (macro) twin variant (i.e. without any movable twin boundary) with the $c$-axis along the shortest dimension. This is automatically secured after compression and it can be easily done in magnetizing cycle as described in experimental chapter.

Despite using detailed and complex method for the evaluation of lattice parameters in the single crystal, the analysis of lattice parameters did not reveal any significant tendencies with load. Observed changes were on the edge of measurement error and, moreover, the tendencies were not consistent. The limitation or inadequacy can arise mainly from strong peak broadening and complex twinned microstructure. Apparently, the used method was still not precise enough or adequate for this kind 


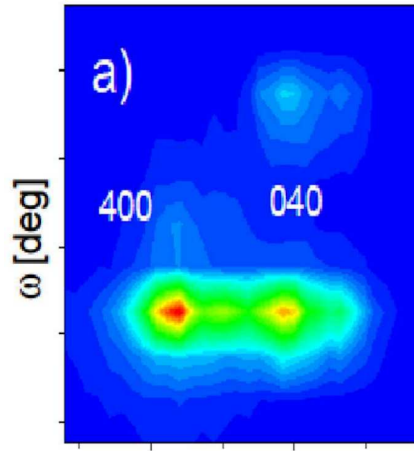

73.5

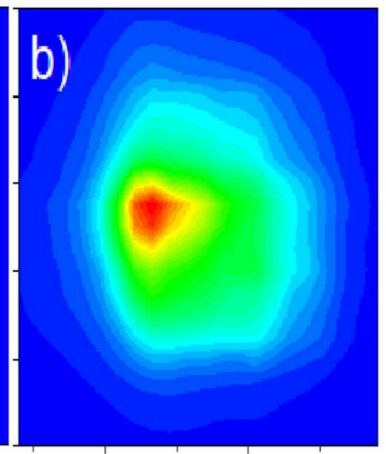

73.5

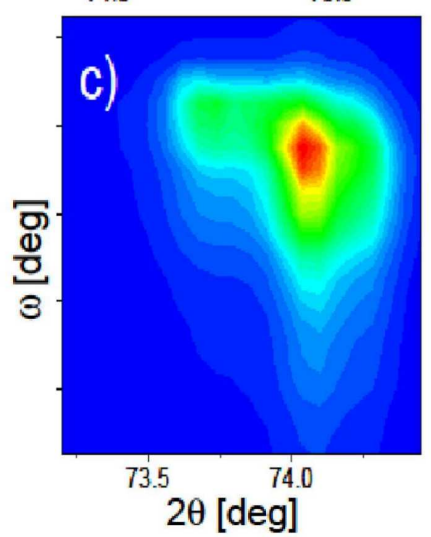

$2 \theta$ [deg]
Fig. 4. 2D $(2 \theta-\omega)$ map of (400) and (040) diffraction peaks; a) initial state, b) immediately after compression, c) after magnetically induced reorientation and magnetizing back to initial orientation. The exact position of $\omega$ depends on sample mounting and thus only marking with distance of $0.2^{\circ}$ is shown. Fine peak splitting in $2 \theta$ is due to presence of $\mathrm{K} \alpha_{1}$ and $\mathrm{K} \alpha_{2}$ lines.

of analysis. More powerful X-ray source, as synchrotron radiation, would be preferred. However, although the structure did not change a lot in agreement with the stress-strain curve, the changes in microstructure were apparent, at least for the highest compression stress.

Figure 4 shows 2D diffraction of (400) and (040) planes in initial state and after the highest stress. Already in initial state the diffraction peaks were broad, which suggests that the coherent area from which the diffraction originated is of submicron size. After compression the broadness of the peaks increased even more both in $2 \theta$ and $\omega$ and slightly shifted. The shift is not, due to large broadening, very significant. The origin of the increased broadening in $2 \theta$ might be due to increased concentration of low angle boundaries (the decrease of the coherent diffraction volume) or the increase of the internal stress. The broadening in $\omega$ indicates that the orientation of $a$ and $b$ lattice vectors continuously varied or the (100) type planes were deformed by compression. After repeated magnetizing the peak became somehow narrower in $\omega$ indicating a reversible change of the microstructure.

The diffraction map indicates that, although there was only one macrotwin variant, i.e. $c$-axis variant, it always consisted of $a-b$ twinning (simultaneous presence of both

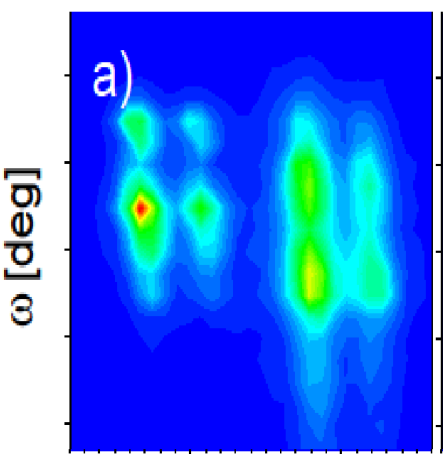

116

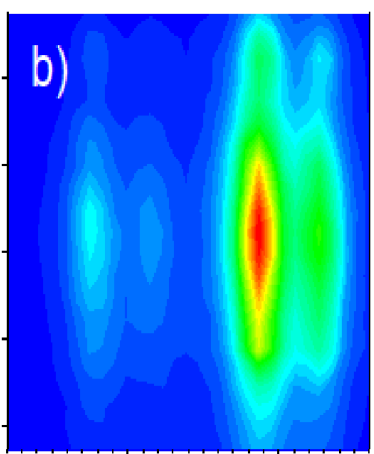

116

117

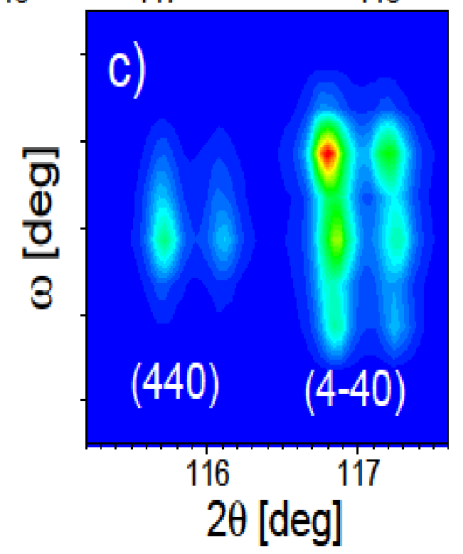

Fig. 5. 2D $(2 \theta-\omega)$ maps of (440) and (4-40) peaks; a) initial state, b) after compression, c) after magnetically induced reorientation and magnetizing back to initial orientation. The exact position of $\omega$ depends on sample mounting and thus only marking with distance of $0.2^{\circ}$ is shown. The peak splitting in $2 \theta$ is due to presence of $\mathrm{K} \alpha_{1}$ and $\mathrm{K} \alpha_{2}$ lines.

planes). The ratio of $a / b$ orientation or twinning changed after compression and repeated magnetizing. Unfortunately we cannot say precisely how much the ratio varied, as the X-ray diffraction was not obtained from the exactly same spot. From previous studies, it is known that ratio $a / b$ can locally vary strongly $[9,10]$. Additionally, the splitting of (440) peak along $2 \theta$ shown in Fig. 5 demonstrates that the $c$-axis macrotwin also containedmonoclinic twining [20]. This twinning too, persisted up to the highest load.

The distinct split of the peaks along $\omega$ axis indicates the initial presence of mosaic blocks. Three of them can be identified. This is in agreement with the previous observation [9] suggesting size of the blocks to be about $0.5 \mathrm{~mm}$. These blocks persisted in low compressive load as individual peaks could be identified in the maps. However, with the increasing stress, the peaks merged and only one very broad peak was apparent in unloaded sample (Fig. 5b). This suggests that whole sample microstructure was deformed (e.g. the planes were bent) and the large stress wiped out the sharp boundary of mosaic blocks. Surprisingly, the mosaic block or at least its coherence somehow recovered after repeated magnetizing in magnetic field. This observation seems to suggest how 
magnetic training can improve the MIR by changing the microstructure.

\section{Conclusion}

Up to $550 \mathrm{MPa}$, the single variant of the $10 \mathrm{M}$ martensite of $\mathrm{Ni}-\mathrm{Mn}-\mathrm{Ga}$ deformed elastically in compression along $c$-axis and after unloading no apparent structure changes were observed by X-ray diffraction. Moreover, the compression did not wipe out the internal structure of the macrotwin variant. The $a-b$ and monoclinic twinning persisted up to the highest load. Despite of small changes in microstructure with the increasing compression stress the mobility of twin boundary decreased, which ultimately could lead to the suppression of MIR. The extrapolation of nucleation field and transforming volume suggested that MIR might cease in well-trained free sample after compression to $1000 \mathrm{MPa}$.

\section{Acknowledgments}

The work was supported by Czech Science Foundation grant to support excellence in research No. 14-36566G.

\section{References}

[1] K. Ullakko, J.K. Huang, C. Kanter, V.V. Kokorin, R.C. O'Handley, Appl. Phys. Lett. 69, 1966 (1996).

[2] A. Sozinov, N. Lanska, A. Soroka, W. Zou, Appl. Phys. Lett. 102, 021902 (2013).

[3] O. Heczko, N. Scheerbaum, O. Gutfleisch, in: Nanoscale Magnetic Materials and Applications, Eds. J.P. Liu, E. Fullerton, O. Gutfleisch, D.J. Sellmyer, Springer Science+Business Media, New York 2009, p. 399.

[4] J. Kopecek, F. Yokaichiya, F. Laufek, M. Jarosová, K. Jurek, J. Drahokoupil, S. Sedláková-Ignácová, P. Molnár, O. Heczko, Acta Phys. Pol. A 122, 475 (2012).
[5] R.C. O'Handley, S.M. Allen, in: Encyclopedia of Smart Materials, Ed. M. Schwartz, Wiley, New York 2001, p. 936.

[6] N. Lanska, O. Söderberg, A. Sozinov, Y. Ge, K. Ullakko, V.K. Lindroos, J. Appl. Phys. 95, 8074 (2004).

[7] A. Sozinov, A.A. Likhachev, N. Lanska, K. Ullakko, Appl. Phys. Lett. 80, 1746 (2002).

[8] S. Kaufmann, R. Niemann, T. Thersleff, U.K. Roessler, O. Heczko, J. Buschbeck, B. Holzapfel, L. Schultz, S. Faehler, New J. Phys. 13, 053029 (2011).

[9] L. Straka, O. Heczko, H. Seiner, N. Lanska, J. Drahokoupil, A. Soroka, S. Faehler, H. Hanninen, A. Sozinov, Acta Mater. 59, 7450 (2011).

[10] O. Heczko, L. Straka, H. Seiner, Acta Mater. 61, 622 (2013).

[11] H. Seiner, L. Straka, O. Heczko, J. Mech. Phys. Solids 64, 198 (2014).

[12] L. Straka, H. Hanninen, O. Heczko, Appl. Phys. Lett. 98, 141902 (2011).

[13] O. Soderberg, I. Aaltio, Y. Ge, O. Heczko, S.-P. Hannula, Mater. Sci. Eng. A 481-482, 80 (2008).

[14] M. Chmielus, K. Rolfs, R. Wimpory, W. Reimers, P. Müllner, R. Schneider, Acta Mater. 58, 3952 (2010).

[15] L. Straka, O. Heczko, H. Haenninen, Acta Mater. 56, 5492 (2008).

[16] N. Scheerbaum, O. Heczko, J. Liu, D. Hinz, L. Schultz, O. Gutfleisch, New J. Phys. 10, 073002 (2008).

[17] O. Heczko, A. Soroka, S.-P. Hannula, Appl. Phys. Lett. 93, 022503 (2008).

[18] Y.L. Ge, O. Heczko, O. Soderberg, S.P. Hannula, Scr. Mater. 54, 2155 (2006).

[19] P.J. Webster, Contemp. Phys. 10, 559 (1969).

[20] O. Heczko, IEEE Trans. Magn. 50, 5807 (2014). 